\title{
Sum Complexes—a New Family of Hypertrees
}

\author{
N. Linial • R. Meshulam • M. Rosenthal
}

Received: 7 March 2009 / Accepted: 2 February 2010 / Published online: 3 March 2010

(C) Springer Science+Business Media, LLC 2010

\begin{abstract}
A $k$-dimensional hypertree $X$ is a $k$-dimensional complex on $n$ vertices with a full $(k-1)$-dimensional skeleton and $\left(\begin{array}{c}n-1 \\ k\end{array}\right)$ facets such that $H_{k}(X ; \mathbb{Q})=0$. Here we introduce the following family of simplicial complexes. Let $n, k$ be integers with $k+1$ and $n$ relatively prime, and let $A$ be a $(k+1)$-element subset of the cyclic group $\mathbb{Z}_{n}$. The sum complex $X_{A}$ is the pure $k$-dimensional complex on the vertex set $\mathbb{Z}_{n}$ whose facets are $\sigma \subset \mathbb{Z}_{n}$ such that $|\sigma|=k+1$ and $\sum_{x \in \sigma} x \in A$. It is shown that if $n$ is prime, then the complex $X_{A}$ is a $k$-hypertree for every choice of $A$. On the other hand, for $n$ prime, $X_{A}$ is $k$-collapsible iff $A$ is an arithmetic progression in $\mathbb{Z}_{n}$.
\end{abstract}

Keywords Hypertrees $\cdot$ Homology $\cdot$ Fourier transform

\section{Introduction}

What is the high-dimensional analogue of a tree? Several approaches to this question can be found in the literature. Here we follow the lead of Kalai [1]. We start with some standard notation. All simplicial complexes we consider $X$ have $n$ vertices, and we always identify the vertex set of $X$ with the cyclic group $\mathbb{Z}_{n}$. The number of $i$-dimensional faces of $X$ is denoted by $f_{i}(X)$. We denote by $\Delta_{n-1}$ the $(n-1)$ -

N. Linial and R. Meshulam are supported by ISF and BSF grants.

N. Linial · M. Rosenthal

Department of Computer Science, Hebrew University, Jerusalem 91904, Israel

N. Linial

e-mail: nati@cs.huji.ac.il

M. Rosenthal

e-mail: mishael40@gmail.com

R. Meshulam ( $\varangle)$

Department of Mathematics, Technion, Haifa 32000, Israel

e-mail: meshulam@math.technion.ac.il 


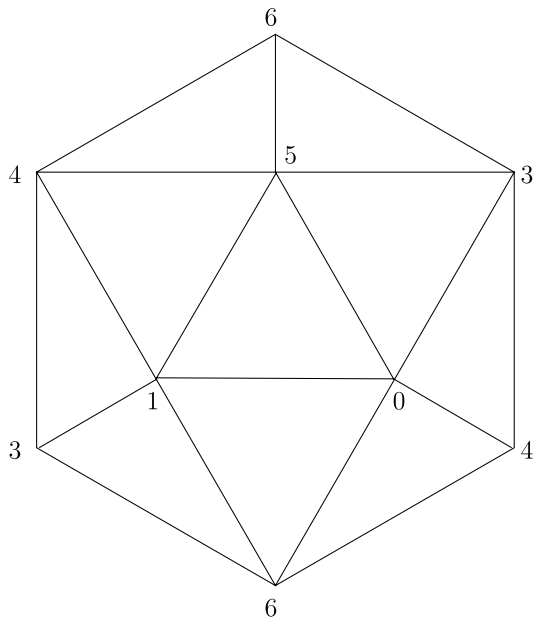

(a) The 6-point triangulation of $\mathbb{R} \mathbb{P}^{2}$

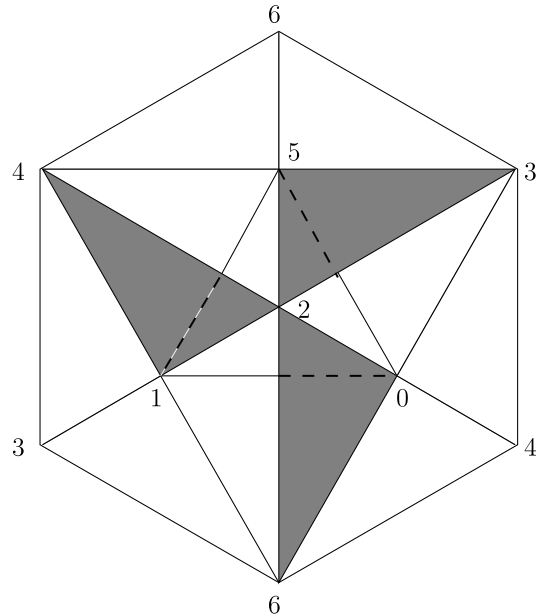

(b) $X_{A}$ for $A=\{0,1,3\} \subset \mathbb{Z}_{7}$

Fig. 1

simplex on the vertex set $\mathbb{Z}_{n}$ and by $\Delta_{n-1}^{(i)}$ the $i$-dimensional skeleton of $\Delta_{n-1}$. A $k$-hypertree is a simplicial complex $\Delta_{n-1}^{(k-1)} \subset X \subset \Delta_{n-1}^{(k)}$ such that $f_{k}(X)=\left(\begin{array}{c}n-1 \\ k\end{array}\right)$ and with a vanishing $k$ th rational homology $H_{k}(X ; \mathbb{Q})=0$. Throughout the paper we assume that $k+1$ is coprime to $n$. For $a \in \mathbb{Z}_{n}$, let $X_{a}$ be the following collection of subsets of $\mathbb{Z}_{n}$ :

$$
X_{a}=\left\{\sigma \subset \mathbb{Z}_{n}:|\sigma|=k+1, \sum_{x \in \sigma} x=a\right\} .
$$

For a subset $A \subset \mathbb{Z}_{n}$ of cardinality $k+1$, define the Sum Complex $X_{A}$ by

$$
X_{A}=\Delta_{n-1}^{(k-1)} \cup\left(\bigcup_{a \in A} X_{a}\right) \text {. }
$$

Example Let $n=7, k=2$, and $A=\{0,1,3\} \subset \mathbb{Z}_{7}$. The two-dimensional complex $X_{A}$ (Fig. 1b) is obtained from the standard 6-point triangulation of the real projective plane $\mathbb{R} \mathbb{P}^{2}$ on the vertices $\{0,1,3,4,5,6\}$ (Fig. 1a) by replacing the face $\{0,1,5\}$ with the three faces $\{0,1,2\},\{0,2,5\}$, and $\{1,2,5\}$ and adding the faces $\{2,3,5\},\{0,2,6\}$, and $\{1,2,4\} . X_{A}$ is clearly homotopy equivalent to $\mathbb{R P}^{2}$.

In this paper we are concerned with topological and combinatorial properties of $X_{A}$. Let $\mathbb{F}$ be a field, and let $h_{i}\left(X_{A} ; \mathbb{F}\right)=\operatorname{dim}_{\mathbb{F}} H_{i}\left(X_{A} ; \mathbb{F}\right)$. Since $X_{A} \supset \Delta_{n-1}^{(k-1)}$, it follows that $h_{0}\left(X_{A} ; \mathbb{F}\right)=1$ and $h_{i}\left(X_{A} ; \mathbb{F}\right)=0$ for $1 \leq i \leq k-2$. Since $k+1$ is coprime to $n$, it follows that for any $y \in \mathbb{Z}_{n}$, the number of $\sigma \subset \mathbb{Z}_{n}$ of cardinality $k+1$ that satisfy $\sum_{x \in \sigma} x=y$ is $\frac{1}{n}\left(\begin{array}{c}n \\ k+1\end{array}\right)$. Therefore $f_{k}\left(X_{A}\right)=\frac{k+1}{n}\left(\begin{array}{c}n \\ k+1\end{array}\right)=\left(\begin{array}{c}n-1 \\ k\end{array}\right)$. The Euler-Poincaré relation $\sum_{i \geq 0}(-1)^{i} f_{i}\left(X_{A}\right)=\sum_{i \geq 0}(-1)^{i} h_{i}\left(X_{A} ; \mathbb{F}\right)$ then implies that $h_{k-1}\left(X_{A} ; \mathbb{F}\right)=h_{k}\left(X_{A} ; \mathbb{F}\right)$. In the sequel we assume that the characteristic of $\mathbb{F}$ does not divide $n$. 
Let $\omega$ be a fixed primitive $n$th root of unity in the algebraic closure $\overline{\mathbb{F}}$. For $x \in \mathbb{Z}_{n}$, let $e(x)=\omega^{x}$. The $n \times n$ Fourier matrix $M$ over $\overline{\mathbb{F}}$ is given by $M(u, v)=e(-u v)$ for $u, v \in \mathbb{Z}_{n}$. For a subset $B \subset \mathbb{Z}_{n}$ of cardinality $k+1$, let $M_{A, B}$ denote the $(k+1) \times$ $(k+1)$ submatrix of $M$ determined by $A$ and $B$. Let $\mathcal{B}_{n, k}$ denote the family of all $(k+1)$-element subsets of $\mathbb{Z}_{n}$ that contain 0 .

\section{Theorem 1.1}

$$
h_{k-1}\left(X_{A} ; \mathbb{F}\right)=h_{k}\left(X_{A} ; \mathbb{F}\right)=\frac{1}{k+1} \sum_{B \in \mathcal{B}_{n, k}} \operatorname{dim} \operatorname{ker} M_{A, B} .
$$

The Fourier transform matrix $M=\left(M_{u v}\right)$ of $\mathbb{Z}_{n}$ over $\overline{\mathbb{Q}} \subset \mathbb{C}$ is given by $M_{u v}=$ $\exp (-2 \pi i u v / n)$. A classical result of Chebotarëv (see, e.g., [3]) asserts that if $n$ is prime, then any square submatrix of $M$ is nonsingular. Theorem 1.1 therefore implies the following:

Corollary 1.2 If $n$ is prime, then $X_{A}$ is a $k$-hypertree.

If $A$ is an arithmetic progression in $\mathbb{Z}_{n}$, then $M_{A, B}$ is a Vandermonde matrix for all $B \in \mathcal{B}_{n, k}$. Hence, by Theorem $1.1, X_{A}$ is $\mathbb{F}$-acyclic for any $\mathbb{F}$ whose characteristic is coprime to $n$. More is in fact true. Let $\sigma$ be a face of dimension at most $k-1$ of a simplicial complex $X$ which is contained in a unique maximal face $\tau$ of $X$, and let $[\sigma, \tau]=\{\eta: \sigma \subset \eta \subset \tau\}$. The operation $X \rightarrow Y=X-[\sigma, \tau]$ is called an elementary $k$-collapse. $X$ is $k$-collapsible if there exists a sequence of elementary $k$-collapses

$$
X=X_{1} \rightarrow X_{2} \rightarrow \cdots \rightarrow X_{m}=\{\emptyset\} .
$$

Note that if $\Delta_{n-1}^{(k-1)} \subset X \subset \Delta_{n-1}^{(k)}$ is $k$-collapsible and $f_{k}(X)=\left(\begin{array}{c}n-1 \\ k\end{array}\right)$, then $X$ is $\mathbb{Z}$-acyclic.

Theorem 1.3 Let $n$ be a prime, and let $A$ be a subset of $\mathbb{Z}_{n}$ of cardinality $k+1$. Then $X_{A}$ is $k$-collapsible iff $A$ is an arithmetic progression in $\mathbb{Z}_{n}$.

Theorems 1.1 and 1.3 are proved in Sects. 2 and 3. In Sect. 4 we compute the homology of $X_{A}$ for $A=\{0,1,3\}$. We conclude in Sect. 5 with some remarks concerning possible extensions and open problems.

\section{Homology of $X_{A}$}

We first recall some topological terminology (see, e.g., [2]). Let $X$ be a finite simplicial complex on the vertex set $V$. For a set $S$ and a field $\mathbb{K}$, let $\mathcal{L}(S, \mathbb{K})$ denote the $\mathbb{K}$-linear space of all $\mathbb{K}$-valued functions on $S$. The space $C^{m}(X ; \mathbb{K})$ of $\mathbb{K}$-valued $m$-cochains of $X$ consists of all functions $\phi \in \mathcal{L}\left(V^{m+1}, \mathbb{K}\right)$ such that $\phi\left(v_{0}, \ldots, v_{m}\right)=\operatorname{sgn}(\pi) \phi\left(v_{\pi(0)}, \ldots, v_{\pi(m)}\right)$ for any permutation $\pi$ on $\{0, \ldots, m\}$ and such that $\phi\left(v_{0}, \ldots, v_{m}\right)=0$ if $\left\{v_{0}, \ldots, v_{m}\right\}$ is not an $m$-dimensional simplex of $X$. 
(In particular, $\phi\left(v_{0}, \ldots, v_{m}\right)=0$ if $v_{i}=v_{j}$ for some $i \neq j$.) The coboundary operator $d_{m}: C^{m}(X ; \mathbb{K}) \rightarrow C^{m+1}(X ; \mathbb{K})$ is given by

$$
d_{m} \phi\left(v_{0}, \ldots, v_{m+1}\right)=\sum_{i=0}^{m+1}(-1)^{i} \phi\left(v_{0}, \ldots, \hat{v}_{i}, \ldots, v_{m+1}\right) .
$$

Let $Z^{m}(X ; \mathbb{K})=\operatorname{ker} d_{m}$ denote the space of $m$-cocycles of $X$ over $\mathbb{K}$, and let $B^{m}(X ; \mathbb{K})=\operatorname{Im} d_{m-1}$ denote the space of $m$-coboundaries of $X$ over $\mathbb{K}$. The $m$-dimensional cohomology space of $X$ with coefficients in $\mathbb{K}$ is

$$
H^{m}(X ; \mathbb{K})=\frac{Z^{m}(X ; \mathbb{K})}{B^{m}(X ; \mathbb{K})}
$$

Let $h^{m}(X, \mathbb{K})=\operatorname{dim}_{\mathbb{K}} H^{m}(X ; \mathbb{K})$. Then $h^{m}(X, \mathbb{K})=h^{m}(X, \mathbb{F})=h_{m}(X ; \mathbb{F})$ for any algebraic extension $\mathbb{K}$ of $\mathbb{F}$. In order to establish Theorem 1.1, we may therefore assume that $\mathbb{F}$ already contains a primitive $n$th root of unity $\omega$.

The Fourier transform of a function $\phi \in \mathcal{L}\left(\mathbb{Z}_{n}^{k} ; \mathbb{F}\right)$ is the function $\mathcal{F}(\phi)=\widehat{\phi} \in$ $\mathcal{L}\left(\mathbb{Z}_{n}^{k} ; \mathbb{F}\right)$ given by

$$
\widehat{\phi}\left(u_{1}, \ldots, u_{k}\right)=\sum_{\left(x_{1}, \ldots, x_{k}\right) \in \mathbb{Z}_{n}^{k}} \phi\left(x_{1}, \ldots, x_{k}\right) e\left(-\sum_{j=1}^{k} u_{j} x_{j}\right) .
$$

The Fourier transform is an automorphism of $\mathcal{L}\left(\mathbb{Z}_{n}^{k} ; \mathbb{F}\right)$.

The proof of Theorem 1.1 involves computing the image of $H^{k-1}(X ; \mathbb{F})$ under the Fourier transform. We first consider the Fourier image of the $(k-1)$-coboundaries.

\section{Claim 2.1}

$$
\mathcal{F}\left(B^{k-1}\left(X_{A} ; \mathbb{F}\right)\right)=\left\{g \in C^{k-1}\left(X_{A} ; \mathbb{F}\right): \operatorname{support}(g) \subset \mathbb{Z}_{n}^{k}-\left(\mathbb{Z}_{n}-\{0\}\right)^{k}\right\}
$$

Proof Let $\psi \in C^{k-2}\left(X_{A} ; \mathbb{F}\right)$. Then

$$
\begin{aligned}
\widehat{d_{k-2} \psi}\left(u_{1}, \ldots, u_{k}\right) \\
=\sum_{\left(x_{1}, \ldots, x_{k}\right) \in \mathbb{Z}_{n}^{k}} d_{k-2} \psi\left(x_{1}, \ldots, x_{k}\right) e\left(-\sum_{j=1}^{k} u_{j} x_{j}\right) \\
=\sum_{\left(x_{1}, \ldots, x_{k}\right) \in \mathbb{Z}_{n}^{k}}\left(\sum_{i=1}^{k}(-1)^{i+1} \psi\left(x_{1}, \ldots, \hat{x}_{i}, \ldots, x_{k}\right)\right) e\left(-\sum_{j=1}^{k} u_{j} x_{j}\right) \\
=\sum_{i=1}^{k}(-1)^{i+1} \sum_{x_{i}} e\left(-u_{i} x_{i}\right) \sum_{x_{1}, \ldots, \hat{x}_{i}, \ldots, x_{k}} \psi\left(x_{1}, \ldots, \hat{x}_{i}, \ldots, x_{k}\right) e\left(-\sum_{j \neq i} u_{j} x_{j}\right) \\
=n \sum_{i=1}^{k}(-1)^{i+1} \delta\left(0, u_{i}\right) \sum_{x_{1}, \ldots, \hat{x}_{i}, \ldots, x_{k}} \psi\left(x_{1}, \ldots, \hat{x}_{i}, \ldots, x_{k}\right) e\left(-\sum_{j \neq i} u_{j} x_{j}\right),
\end{aligned}
$$


where $\delta\left(0, u_{i}\right)=1$ if $u_{i}=0$ and is zero otherwise. Therefore,

$$
\mathcal{F}\left(B^{k-1}\left(X_{A} ; \mathbb{F}\right)\right) \subset\left\{g \in C^{k-1}\left(X_{A} ; \mathbb{F}\right): \operatorname{support}(g) \subset \mathbb{Z}_{n}^{k}-\left(\mathbb{Z}_{n}-\{0\}\right)^{k}\right\}
$$

Equality follows since both spaces have dimension $\left(\begin{array}{l}n-1 \\ k-1\end{array}\right)$ over $\mathbb{F}$.

We next study the Fourier image of the $(k-1)$-cocycles of $X_{A}$. Fix a $\phi \in$ $C^{k-1}\left(X_{A} ; \mathbb{F}\right)$. For $a \in \mathbb{Z}_{n}$, define the function $f_{a} \in \mathcal{L}\left(\mathbb{Z}_{n}^{k} ; \mathbb{F}\right)$ by

$$
\begin{aligned}
f_{a}\left(x_{1}, \ldots, x_{k}\right) & =d_{k-1} \phi\left(a-\sum_{i=1}^{k} x_{i}, x_{1}, \ldots, x_{k}\right) \\
& =\phi\left(x_{1}, \ldots, x_{k}\right)+\sum_{i=1}^{k}(-1)^{i} \phi\left(a-\sum_{j=1}^{k} x_{j}, x_{1}, \ldots, \hat{x}_{i}, \ldots, x_{k}\right) .
\end{aligned}
$$

Let $T$ be the automorphism of $\mathbb{Z}_{n}^{k}$ given by

$$
T\left(u_{1}, \ldots, u_{k}\right)=\left(u_{2}-u_{1}, \ldots, u_{k}-u_{1},-u_{1}\right) .
$$

Then $T^{k+1}=I$ and, for $1 \leq i \leq k$,

$$
T^{i}\left(u_{1}, \ldots, u_{k}\right)=\left(u_{i+1}-u_{i}, \ldots, u_{k}-u_{i},-u_{i}, u_{1}-u_{i}, \ldots, u_{i-1}-u_{i}\right) .
$$

Claim 2.2 Let $u=\left(u_{1}, \ldots, u_{k}\right) \in \mathbb{Z}_{n}^{k}$. Then

$$
\widehat{f}_{a}(u)=\widehat{\phi}(u)+\sum_{i=1}^{k}(-1)^{k i} e\left(-u_{i} a\right) \widehat{\phi}\left(T^{i} u\right) .
$$

Proof For $1 \leq i \leq k$, let $\psi_{i} \in \mathcal{L}\left(\mathbb{Z}_{n}^{k}, \mathbb{F}\right)$ be given by

$$
\psi_{i}\left(x_{1}, \ldots, x_{k}\right)=\phi\left(a-\sum_{j=1}^{k} x_{j}, x_{1}, \ldots, \hat{x}_{i}, \ldots, x_{k}\right) .
$$

Then

$$
\widehat{\psi}_{i}(u)=\sum_{\left(x_{1}, \ldots, x_{k}\right) \in \mathbb{Z}_{n}^{k}} \phi\left(a-\sum_{j=1}^{k} x_{j}, x_{1}, \ldots, \hat{x}_{i}, \ldots, x_{k}\right) e\left(-\sum_{j=1}^{k} u_{j} x_{j}\right) .
$$

Substituting

$$
y_{j}= \begin{cases}a-\sum_{\ell=1}^{k} x_{\ell}, & j=1 \\ x_{j-1}, & 2 \leq j \leq i \\ x_{j}, & i+1 \leq j \leq k\end{cases}
$$


it follows that

$$
\sum_{j=1}^{k} u_{j} x_{j}=\left(a-y_{1}\right) u_{i}+\sum_{j=2}^{i}\left(u_{j-1}-u_{i}\right) y_{j}+\sum_{j=i+1}^{k}\left(u_{j}-u_{i}\right) y_{j} .
$$

Therefore,

$$
\begin{aligned}
\widehat{\psi}_{i}(u)= & e\left(-u_{i} a\right) \\
& \times \sum_{y=\left(y_{1}, \ldots, y_{k}\right) \in \mathbb{Z}_{n}^{k}} \phi(y) e\left(u_{i} y_{1}-\sum_{j=2}^{i}\left(u_{j-1}-u_{i}\right) y_{j}-\sum_{j=i+1}^{k}\left(u_{j}-u_{i}\right) y_{j}\right) \\
= & e\left(-u_{i} a\right) \widehat{\phi}\left(-u_{i}, u_{1}-u_{i}, \ldots, u_{i-1}-u_{i}, u_{i+1}-u_{i}, \ldots, u_{k}-u_{i}\right) \\
= & e\left(-u_{i} a\right)(-1)^{i(k-i)} \widehat{\phi}\left(T^{i} u\right) .
\end{aligned}
$$

Now (2) follows from (3) since $f_{a}=\phi+\sum_{i=1}^{k}(-1)^{i} \psi_{i}$.

For $u \in \mathbb{Z}_{n}^{k}$, let $E_{u}=\left\{T^{i} u: 0 \leq i \leq k\right\}$, and let

$$
L_{u}=\bigcap_{a \in A}\left\{g \in \mathcal{L}\left(E_{u}, \mathbb{F}\right): g(u)+\sum_{i=1}^{k}(-1)^{k i} e\left(-u_{i} a\right) g\left(T^{i} u\right)=0\right\} .
$$

Let $\phi \in Z^{k-1}\left(X_{A} ; \mathbb{F}\right)$. Then, for all $a \in A$ and $\left(x_{1}, \ldots, x_{k}\right) \in \mathbb{Z}_{n}^{k}$,

$$
f_{a}\left(x_{1}, \ldots, x_{k}\right)=d_{k-1} \phi\left(a-\sum_{i=1}^{k} x_{i}, x_{1}, \ldots, x_{k}\right)=0 .
$$

Equation (2) then implies that, for all $a \in A$ and $u \in \mathbb{Z}_{n}^{k}$,

$$
\widehat{\phi}(u)+\sum_{i=1}^{k}(-1)^{k i} e\left(-u_{i} a\right) \widehat{\phi}\left(T^{i} u\right)=0 .
$$

Writing $\widehat{\phi}_{\mid E_{u}}$ for the restriction of $\widehat{\phi}$ to $E_{u}$, we obtain the following:

Corollary 2.3 Let $\phi \in C^{k-1}\left(X_{A} ; \mathbb{F}\right)$. Then $\phi \in Z^{k-1}\left(X_{A} ; \mathbb{F}\right)$ iff $\widehat{\phi}_{\mid E_{u}} \in L_{u}$ for all $u \in \mathbb{Z}_{n}^{k}$.

Let the symmetric group $S_{k}$ act on $\mathbb{Z}_{n}^{k}$ by

$$
\sigma\left(\left(u_{1}, \ldots, u_{k}\right)\right)=\left(u_{\sigma^{-1}(1)}, \ldots, u_{\sigma^{-1}(k)}\right),
$$

and let $G_{n, k}$ denote the subgroup of $\operatorname{Aut}\left(\mathbb{Z}_{n}^{k}\right)$ generated by $T$ and $S_{k}$. The subset

$$
D_{n, k}=\left\{\left(u_{1}, \ldots, u_{k}\right) \in\left(\mathbb{Z}_{n}-\{0\}\right)^{k}: u_{i} \neq u_{j} \text { for } i \neq j\right\}
$$


is clearly invariant under $G_{n, k}$.

\section{Claim 2.4}

(i) Let $\sigma \in S_{k}$ and $1 \leq i \leq k$. Then $\eta=T^{i} \sigma T^{-\sigma^{-1}(i)} \in S_{k}$ and $\operatorname{sgn}(\eta)=$ $(-1)^{k\left(i+\sigma^{-1}(i)\right)} \operatorname{sgn}(\sigma)$.

(ii) Any element of $G_{n, k}$ can be written uniquely as $\sigma T^{i}$ where $\sigma \in S_{k}$ and $0 \leq i \leq k$. $G_{n, k}$ acts freely on $D_{n, k}$.

(iii) $L_{u}=L_{T^{j} u}$ for all $u \in D_{n, k}$ and $0 \leq j \leq k$.

\section{Proof}

(i) For $1 \leq \ell \leq k$, let $\tau_{\ell} \in S_{k}$ be given by

$$
\tau_{\ell}(i)= \begin{cases}k-\ell+1+i, & 1 \leq i \leq \ell-1, \\ k-\ell+1, & i=\ell, \\ i-\ell, & \ell+1 \leq i \leq k\end{cases}
$$

It can be checked that

$$
\eta=T^{i} \sigma T^{-\sigma^{-1}(i)}=\tau_{k-i+1}^{-1} \sigma \tau_{k-\sigma^{-1}(i)+1} .
$$

Noting that $\operatorname{sgn}\left(\tau_{\ell}\right)=(-1)^{k \ell+1}$, it thus follows that

$$
\operatorname{sgn}(\eta)=\operatorname{sgn}(\sigma) \operatorname{sgn}\left(\tau_{k-i+1}\right) \operatorname{sgn}\left(\tau_{k-\sigma^{-1}(i)+1}\right)=(-1)^{k\left(i+\sigma^{-1}(i)\right)} \operatorname{sgn}(\sigma) .
$$

(ii) It follows from (i) that

$$
G_{n, k}=\left\{\sigma T^{i}: \sigma \in S_{k}, 0 \leq i \leq k\right\}
$$

Let $u=\left(u_{1}, \ldots, u_{k}\right) \in D_{n, k}$, and let $v=\left(v_{1}, \ldots, v_{k}\right)=\sigma T^{i} u$. If $i \neq 0$, then

$$
\sum_{j=1}^{k} v_{j}=\sum_{j=1}^{k} u_{j}-(k+1) u_{i} \neq \sum_{j=1}^{k} u_{j}
$$

and therefore $\sigma T^{i} u \neq u$. It follows that $G_{n, k}$ acts freely on $D_{n, k}$ and that the representation of an element of $G_{n, k}$ as $\sigma T^{i}$ is unique.

(iii) Let $g \in L_{u}$ and $a \in A$. Then

$$
\begin{aligned}
& g\left(T^{j} u\right)+\sum_{i=1}^{k}(-1)^{i k} e\left(-\left(T^{j} u\right)_{i} a\right) g\left(T^{i+j} u\right) \\
& =g\left(T^{j} u\right)+\sum_{i=1}^{k-j}(-1)^{i k} e\left(-\left(u_{i+j}-u_{j}\right) a\right) g\left(T^{i+j} u\right) \\
& \quad+(-1)^{(k-j+1) k} e\left(u_{j} a\right) g(u)
\end{aligned}
$$




$$
\begin{gathered}
+\sum_{i=k-j+2}^{k}(-1)^{i k} e\left(-\left(u_{i-k+j-1}-u_{j}\right) a\right) g\left(T^{i+j} u\right) \\
=(-1)^{j k} e\left(u_{j} a\right)\left(g(u)+\sum_{i=1}^{k}(-1)^{i k} e\left(-u_{i} a\right) g\left(T^{i} u\right)\right)=0 .
\end{gathered}
$$

Hence $g \in L_{T^{j} u}$.

Proof of Theorem 1.1: Let $R \subset D_{n, k}$ be a fixed set of representatives of the orbits of $G_{n, k}$ on $D_{n, k}$. Then $|R|=\frac{\left|D_{n, k}\right|}{\left|G_{n, k}\right|}=\frac{1}{k+1}\left(\begin{array}{c}n-1 \\ k\end{array}\right)$. Consider the mapping

$$
\Theta: Z^{k-1}\left(X_{A} ; \mathbb{F}\right) \rightarrow \bigoplus_{u \in R} L_{u}
$$

given by

$$
\Theta(\phi)=\left(\widehat{\phi}_{\mid E_{u}}: u \in R\right) .
$$

\section{Claim 2.5}

$$
\operatorname{ker} \Theta=B^{k-1}\left(X_{A} ; \mathbb{F}\right)
$$

\section{Proof}

$$
\begin{aligned}
\operatorname{ker} \Theta & =\left\{\phi \in Z^{k-1}\left(X_{A} ; \mathbb{F}\right): \widehat{\phi}_{\mid E_{u}}=0 \text { for all } u \in R\right\} \\
& =\left\{\phi \in Z^{k-1}\left(X_{A} ; \mathbb{F}\right): \widehat{\phi}(u)=0 \text { for all } u \in D_{n, k}\right\}=B^{k-1}\left(X_{A} ; \mathbb{F}\right)
\end{aligned}
$$

by Claim 2.1 .

Claim 2.6 $\Theta$ is surjective.

Proof Let $\left(g_{u}: u \in R\right) \in \bigoplus_{u \in R} L_{u}$. Define $g \in C^{k-1}\left(X_{A} ; \mathbb{F}\right)$ by

$$
g(v)= \begin{cases}0, & v \notin D_{n, k}, \\ \operatorname{sgn}(\sigma) g_{u}\left(T^{j} u\right), & v=\sigma T^{j} u, \text { where } u \in R .\end{cases}
$$

Clearly $\Theta\left(\mathcal{F}^{-1}(g)\right)=\left(g_{u}: u \in R\right)$. To show that $\mathcal{F}^{-1}(g) \in Z^{k-1}\left(X_{A} ; \mathbb{F}\right)$, it suffices by Corollary 2.3 to check that $g \in L_{v}$ for all $v \in \mathbb{Z}_{n}^{k}$. If $v \notin D_{n, k}$, then $g_{\mid E_{v}}=0$. Suppose then that $v=\sigma T^{j} u \in D_{n, k}$, where $u \in R$ and $0 \leq j \leq k$. Combining Claim 2.4(i) and (5), it follows that

$$
\begin{aligned}
& g(v)+\sum_{i=1}^{k}(-1)^{i k} e\left(-v_{i} a\right) g\left(T^{i} v\right) \\
& =g\left(\sigma T^{j} u\right)+\sum_{i=1}^{k}(-1)^{i k} e\left(-\left(\sigma T^{j} u\right)_{i} a\right) g\left(T^{i} \sigma T^{j} u\right)
\end{aligned}
$$




$$
\begin{aligned}
= & \operatorname{sgn}(\sigma) g_{u}\left(T^{j} u\right) \\
& +\sum_{i=1}^{k}(-1)^{i k} e\left(-\left(T^{j} u\right)_{\sigma^{-1}(i)} a\right)(-1)^{k\left(i+\sigma^{-1}(i)\right)} \operatorname{sgn}(\sigma) g_{u}\left(T^{\sigma^{-1}(i)+j} u\right) \\
= & \operatorname{sgn}(\sigma)\left(g_{u}\left(T^{j} u\right)+\sum_{i=1}^{k}(-1)^{i k} e\left(-\left(T^{j} u\right)_{i} a\right) g_{u}\left(T^{i+j} u\right)\right) \\
= & (-1)^{j k} \operatorname{sgn}(\sigma) e\left(u_{j} a\right)\left(g_{u}(u)+\sum_{i=1}^{k}(-1)^{i k} e\left(-u_{i} a\right) g_{u}\left(T^{i} u\right)\right)=0 .
\end{aligned}
$$

Claims 2.5 and 2.6 imply that

$$
H^{k-1}\left(X_{A}, \mathbb{F}\right) \cong \bigoplus_{u \in R} L_{u} .
$$

For $u=\left(u_{1}, \ldots, u_{k}\right) \in D_{n, k}$, let $B_{u}=\left\{0, u_{1}, \ldots, u_{k}\right\}$. Then $\operatorname{dim} L_{u}=\operatorname{dim} \operatorname{ker} M_{A, B_{u}}$. Combining (6) with Claim 2.4(iii), it thus follows that

$$
\begin{aligned}
h^{k-1}\left(X_{A} ; \mathbb{F}\right) & =\sum_{u \in R} \operatorname{dim} L_{u} \\
& =\frac{1}{k+1} \sum_{u \in R} \sum_{j=0}^{k} \operatorname{dim} L_{T^{j} u}=\frac{1}{k+1} \sum_{B \in \mathcal{B}_{n, k}} \operatorname{dim} \operatorname{ker} M_{A, B} .
\end{aligned}
$$

\section{When is $X_{A}$ Collapsible?}

In this section we prove Theorem 1.3 , so that in this section $n$ is prime. We find it convenient to maintain the vertices in a face sorted according to the order induced from $\mathbb{N}$ and also refer to subsets of $\mathbb{F}_{n}$ as sorted vectors and not only as sets.

\subsection{Equivalence}

Let $\phi: \mathbb{F}_{n} \rightarrow \mathbb{F}_{n}$ be the linear map $\phi(x)=\alpha x+\beta$. It is clear that the image of $X_{a}$ under $\phi$ is $X_{t}$ where $t=\alpha a+(k+1) \beta$. We say that the complexes $X_{a_{0}, \ldots, a_{k}}$ and $X_{b_{0}, \ldots, b_{k}}$ are equivalent iff there exist a permutation $\pi$ on $\left\{b_{0}, \ldots, b_{k}\right\}$ and $\alpha, \beta$ such that $\pi\left(b_{i}\right)=\alpha a_{i}+(k+1) \beta$ for every $0 \leq i \leq k$. Equivalent complexes are clearly isomorphic.

It is an easy observation that $a_{0}, \ldots, a_{k}$ is an arithmetic progression iff $X_{a_{0}, \ldots, a_{k}}$ is equivalent to the complex $X_{0, \ldots, k}$. We show that $X=X_{0, \ldots, k}$ is collapsible, whence $X_{a_{0}, \ldots, a_{k}}$ is collapsible for $a_{0}, \ldots, a_{k}$ an arithmetic progression.

\subsection{Proof of Sufficiency}

To show that $X$ is collapsible, we introduce an order $\prec_{R}$ by which we remove the $k$-faces from $X$. We first need some preliminary definitions. With every $k$-face $u \in X$, 
we associate a vector $h(u)$ of dimension $\left\lceil\frac{k}{2}\right\rceil$. The $i$ th coordinate in $h$ counts how many integers in the interval $\left[u_{i}, u_{k-i}\right]$ do not belong to $\left\{u_{i}, \ldots, u_{k-i}\right\}$. Namely, the $i$ th coordinate of $h(u)$ is

$$
h_{i}(u):=u_{k-i}-u_{i}-(k-2 i) .
$$

Clearly $h_{i}(u)$ is nonincreasing in $i$. For every two $k$-faces $u, v \in X$, we say that $u \prec_{L} v$ if $h(u)$ is lexicographically smaller than $h(v)$. When $h(u)=h(v)$, we say that $u \equiv_{L} v$. It should be clear that $h$ is invariant under set reversal, i.e., $x \rightarrow n-x$. It is also invariant under shifts that "do not overflow" in the obvious sense, but we will not use this fact. If $u \neq_{L} v$ for some $u, v \in X$, we denote by $\delta_{L}(u, v)$ the first index for which $h(u)$ and $h(v)$ differ. Thus if $u \prec_{L} v$ and $\delta_{L}(u, v)=i$, then $h_{j}(u)=h_{j}(v)$ for all $j<i$ and $h_{i}(u)<h_{i}(v)$.

For $i, j \in \mathbb{F}_{n}$, it is convenient to define $\rho(i, j)$ as $i-j$ if $i>j$ and as $j-i$ otherwise. This is extended as usual to $\rho(i, A)=\min \{\rho(i, a) \mid a \in A\}$ and $\rho(A, B)=$ $\min \{\rho(a, b) \mid a \in A, b \in B\}$.

If $u \in X_{i}$ and $v \in X_{j}$, we say that $u \prec_{I} v$ if $i$ is closer than $j$ to $\{0, k\}$, i.e., if $\rho(i,\{0, k\})<\rho(j,\{0, k\})$. We say that $u \equiv_{I} v$ when $\rho(i,\{0, k\})=\rho(j,\{0, k\})$, namely, $i=j$ or $i=k-j$. Letting $i^{\prime}=\rho(i,\{0, k\})$, it is clear that $u \prec_{I} v$ iff $i^{\prime}<j^{\prime}$. If $u \not \equiv I V$, we denote $\delta_{I}(u, v)=\min \left\{i^{\prime}, j^{\prime}\right\}=\rho(\{i, j\},\{0, k\})$.

We are now ready to define the relation $\prec_{R}$. This is done in terms of the relations $\prec_{L}$ and $\prec_{I}$. To begin, $u \equiv_{R} v$ iff $u \equiv_{L} v$ and $u \equiv_{I} v$. If $u \preceq_{L} v$ and $u \preceq_{I} v$ and at least one inequality is proper, then $u \prec_{R} v$. Finally, when $u \prec_{L} v$ and $u \succ_{I} v$, the order $\prec_{R}$ is determined according to the smaller of $\delta_{I}(u, v), \delta_{L}(u, v)$. Namely, if $\delta_{I}(u, v)<\delta_{L}(u, v)$, then $u \succ_{R} v$, and if $\delta_{I}(u, v) \geq \delta_{L}(u, v)$, then $u \prec_{R} v$.

To sum up, for $u, v \in X$ :

1. if $u \equiv_{I} v$ and $u \equiv_{L} v$, then $u \equiv_{R} v$

2. if $u \equiv_{I} v$ and $u \prec_{L} v$, then $u \prec_{R} v$

3. if $u \prec_{I} v$, then $u \prec_{R} v$ unless

(a) $u \succ_{L} v$ and

(b) $\delta_{L}(u, v) \leq \delta_{I}(u, v)$

in which case $u \succ_{R} v$

To clarify this definitions a little bit more, we present an example from the complex $X_{0,1,2,3}$ over $\mathbb{F}_{7}$. Let $u=\{0,1,2,5\}$ and $v=\{1,2,5,6\}$. The set $u$ has two missing integers between 0 and 5 and no missing integers between 1 and 2 ; hence $h(u)=h(\{0,1,2,5\})=(2,0)$. Similarly $h(v)=h(\{1,2,5,6\})=(2,2)$. Also, $u \prec_{L} v$ because $(2,0)$ is lexicographically smaller than $(2,2)$. Furthermore, $\delta_{L}(u, v)=1$ because the first coordinate the vectors differ is the second coordinate (and we start indexing coordinates from zero). Now $u \in X_{1}$ since $0+1+2+5 \equiv 1 \bmod 7$. Similarly $v \in X_{0}$. We next calculate that $1^{\prime}=1=\rho(1,\{0,7\})$ and $0^{\prime}=0$. Hence $v \prec_{I} u$ because $0^{\prime}<1^{\prime}$, and $\delta_{I}(u, v)=\min \{0,1\}=0$. To recap, $u \prec_{L} v$ and $v \prec_{I} u$, so we turn to compare $0=\delta_{I}(u, v)<\delta_{L}(u, v)=1$; it follows that in this case the order $R$ is determined by $I$, and hence $\{0,1,2,5\}=u \succ_{R} v=\{1,2,5,6\}$. A full description of the order $R$ on $X_{0,1,2,3}$ over $\mathbb{F}_{7}$ is shown in Figs. 2 and 3.

A few words are in order about Fig. 2. The rows are sorted by the lexicographic order of $h(\cdot)$. The columns on the right include all facets of $X$ sorted by value of $i^{\prime}$. 


\begin{tabular}{llll}
\hline$\delta_{L}(x, y)$ & $h(x)$ & $i^{\prime}=0$ & $i^{\prime}=1$ \\
\hline 0 & $(0,0)$ & $\{2,3,4,5\},\{1,2,3,4\}$ & \\
1 & $(1,0)$ & $\{0,1,2,4\},\{2,4,5,6\}$ & $\{2,3,4,6\},\{0,2,3,4\}$ \\
0 & $(1,1)$ & & $\{0,1,3,4\},\{2,3,5,6\}$ \\
1 & $(2,0)$ & $\{1,3,4,6\},\{0,2,3,5\}$ & $\{0,1,2,5\},\{1,4,5,6\}$ \\
1 & $(2,1)$ & & $\{1,3,5,6\},\{0,1,3,5\}$ \\
0 & $(2,2)$ & $\{1,2,5,6\},\{0,1,4,5\}$ & \\
1 & $(3,0)$ & & $\{0,4,5,6\},\{0,1,2,6\}$ \\
\hline
\end{tabular}

Fig. $2 X_{0,1,2,3}$ parameters over $\mathbb{F}_{7}$

Fig. 3 The order of collapse determined by $\prec_{R}$

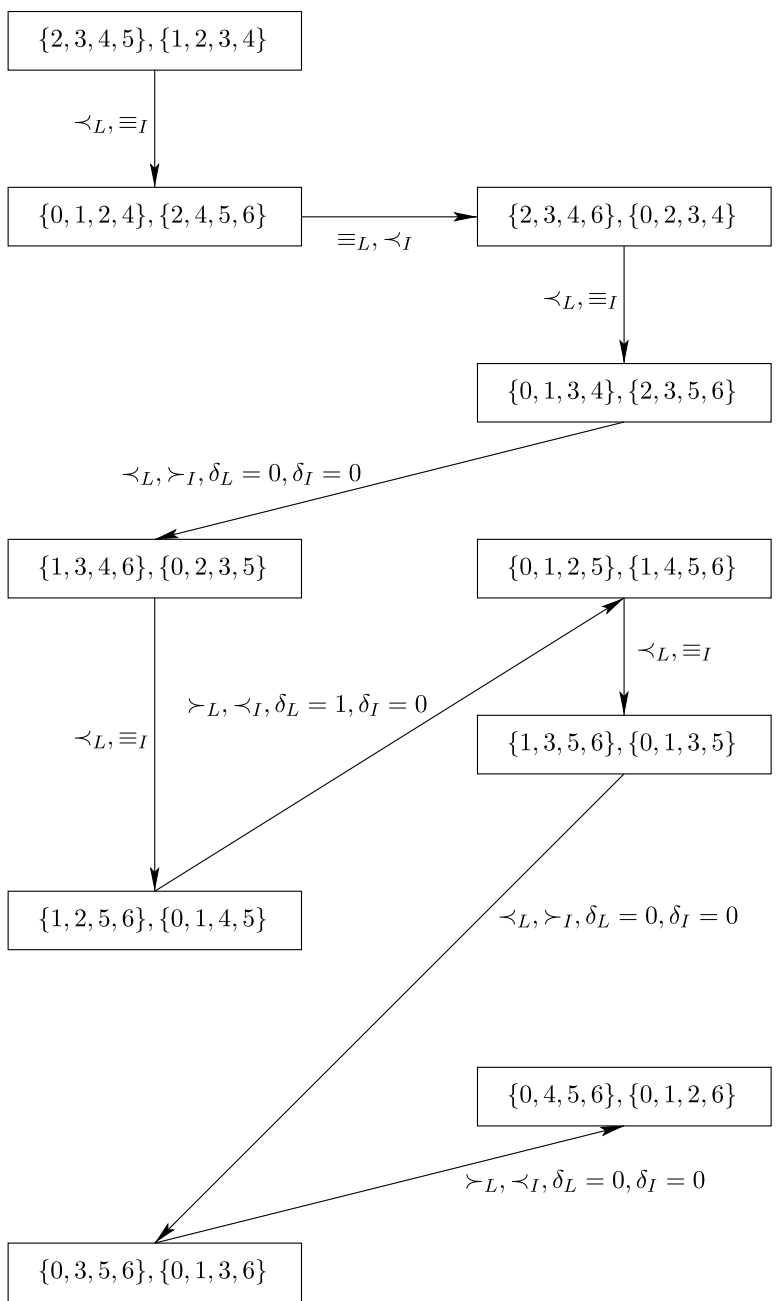


Note that for each value of $h$ and each $i^{\prime}$, there are two facets that attain this pair of values. The leftmost column gives the value of $\delta_{L}(x, y)$ for every two consecutive lines in the table.

We now turn to show that $X$ can indeed be collapsed in the order $\prec_{R}$. That is, for every $x \in X$, it is possible to apply an elementary collapse step to $x$ if all the $\prec R^{-}$ predecessors of $x$ have already been collapsed. In order to show this, we need to point out a free $(k-1)$-face that is contained in $x$. What we will show is that for $x \in X_{a}$, the face $\hat{x}:=x \backslash\left\{x_{a}\right\}$ is free. (Note that since $x \in X=X_{0, \ldots, k}$, there indeed must exist some $a \in\{0, \ldots, k\}$ such that $x \in X_{a}$.) It may be helpful to mention that $a$ plays a double role here. It is an index in the vector $x$ as well as the sum of the elements of $x$. Being free means that all the $k$-faces containing $\hat{x}$ precede $x$ in the order $\prec_{R}$. A $k$-face that contains $\hat{x}$ has the form $y^{(b)}:=\hat{x} \cup\left\{x_{a}+(b-a)\right\}$ with $0 \leq b \leq k$ and $b \neq a$. Clearly, $y^{(b)}$ is a $k$-face in $X$ iff $x_{a}+(b-a) \notin \hat{x}$. Also, in this case $y^{(b)} \in X_{b}$, as we assume below.

The proof that $y^{(b)} \prec_{R} x$ has two cases:

1. We first consider the case where $y^{(b)} \succeq_{I} x$. Since $y^{(b)} \in X_{b}$ and $x \in X_{a}$, the meaning of $y^{(b)} \succeq_{I} x$ is that $b^{\prime} \geq a^{\prime}$. Therefore $\delta_{I}\left(y^{(b)}, x\right)$, which is the smaller of $a^{\prime}$ and $b^{\prime}$, equals $a^{\prime}$. This means that $b$ lies between $a$ and $k-a$ (whether $a$ or $k-a$ is bigger is immaterial here).

- Consequently, $x_{a}+(b-a)$ is in the interval $\left[x_{a^{\prime}}, x_{k-a^{\prime}}\right]$. It follows that the first and last $a^{\prime}-1$ elements of $x$ and $y^{(b)}$ are identical. In particular, $h_{i}\left(y^{(b)}\right)=$ $h_{i}(x)$ for $i<a^{\prime}$.

- We recall that $y^{(b)}$ is created by removing $x_{a}$ from $x$ and replacing it by the term $x_{a}+(b-a)$. Thus the interval $\left[y_{a^{\prime}}^{(b)}, y_{k-a^{\prime}}^{(b)}\right]$ is shorter than $\left[x_{a^{\prime}}, x_{k-a^{\prime}}\right]$. It follows that the first coordinate where $h\left(y^{(b)}\right)$ and $h(x)$ differ is the $a^{\prime}$ th coordinate, where $h_{a^{\prime}}\left(y^{(b)}\right)<h_{a^{\prime}}(x)$. Consequently, $y^{(b)} \prec_{L} x$ and $a^{\prime}=\delta_{L}\left(y^{(b)}, x\right)$.

- If $y^{(b)} \equiv_{I} x$, then we are done, because we already know that $y^{(b)} \prec_{L} x$. By the definition of $\prec_{R}$, this yields the desired conclusion $y^{(b)} \prec_{R} x$.

- If $y^{(b)} \succ_{I} x$, then from the previous points we conclude that $a^{\prime}=\delta_{L}\left(y^{(b)}, x\right)=$ $\delta_{I}\left(y^{(b)}, x\right)$. To sum up, $y^{(b)} \prec_{L} x$ and $\delta_{L}\left(y^{(b)}, x\right)=\delta_{I}\left(y^{(b)}, x\right)$, which yields by definition, $y^{(b)} \prec_{R} x$, as claimed.

2. Now consider the case $y^{(b)} \prec_{I} x$. This means that $b^{\prime}<a^{\prime}$. Therefore $b^{\prime}=$ $\delta_{I}\left(y^{(b)}, x\right)$. Consequently, $b$ does not lie between $a$ and $k-a$.

- It follows that $x_{a}+(b-a) \in\left[x_{b^{\prime}}, x_{k-b^{\prime}}\right]$. Consequently, the first and last $b^{\prime}+1$ elements of $x$ and $y^{(b)}$ are identical. In particular, $h_{i}\left(y^{(b)}\right)=h_{i}(x)$ for $i \leq b^{\prime}$. Thus $\delta_{L}\left(y^{(b)}, x\right)>b^{\prime}$.

- If $y^{(b)} \preceq_{L} x$, then $y^{(b)} \prec_{R} x$, and we are done.

- If $y^{(b)} \succ_{L} x$, then from the previous points we conclude that $b^{\prime}=\delta_{I}\left(y^{(b)}, x\right)<$ $\delta_{L}\left(y^{(b)}, x\right)$. Hence $y^{(b)} \succ_{I} x$ and $\delta_{I}\left(y^{(b)}, x\right)<\delta_{L}\left(y^{(b)}, x\right)$. Again, by definition, $y^{(b)} \prec_{R} x$, as claimed.

This completes the proof that $X_{0, \ldots, k}$ is collapsible and hence that $X=X_{a_{0}, \ldots, a_{k}}$ is collapsible whenever $a_{0}, \ldots, a_{k}$ is an arithmetic progression. 


\subsection{Proof of Necessity}

We now turn to show that if $a_{0}, \ldots, a_{k}$ is not arithmetic, then $X_{a_{0}, \ldots, a_{k}}$ is not collapsible. In fact we show that in this case exactly $k+1$ elementary collapse steps can be carried out.

For $X \subseteq \mathbb{F}_{n}$, we denote as usual by $X+a$ the $a$-shift of $X$, namely, the set $\{x+a \mid x \in X\}$. We start with the following simple observation.

Observation 3.1 Let $n$ be a prime. A subset $X \subsetneq \mathbb{F}_{n}$ is an arithmetic progression iff there is an element l for which $|(X+l) \backslash X|=1$.

When is the $(k-1)$-face $x_{1}, \ldots, x_{k}$ a free face? This is the case iff, for each $k \geq i \geq 1$, the element $x_{i}+\sum_{j=1}^{k} x_{j}$ belongs to the set $\left\{a_{0}, \ldots, a_{k}\right\}$. If $x_{i}+\sum x_{j}=a_{l}$, this means that $x_{1}, \ldots, x_{k}$ cannot be extended to a $k$-face in $X_{a_{l}}$. This translates into a linear system of equations in $x_{1}, \ldots, x_{k}$ whose matrix has 2 's along the main diagonal and 1's elsewhere. Such a matrix is nonsingular, so the solution is unique. Also, all the $k$ terms $x_{i}+\sum x_{j}$ are distinct, so the only choice we have in constructing this linear system is which of the $k+1$ elements in $\left\{a_{0}, \ldots, a_{k}\right\}$ to omit. There are $k+1$ such choices which yield $k+1$ distinct collapse steps that can be carried out.

We now explicitly describe the $k+1$ collapse steps that can be carried out. Each of these collapsible faces has the form $x^{(t)}:=\left\{a_{0}+l_{t}, \ldots, a_{k}+l_{t}\right\} \in X_{a_{t}}$ for some $l_{0}, \ldots l_{k}$.

The condition $x^{(t)} \in X_{a_{t}}$ determines $l_{t}$ via $l_{t}=\frac{a_{t}-\sum_{i=0}^{k} a_{i}}{k+1}$. We claim that the face $y:=x^{(t)} \backslash\left\{a_{t}+l_{t}\right\}$ is free. The sum of $y$ 's elements is $-l_{t}$, so that for every $i \neq t$, we need to add the term $\left\{a_{i}+l_{t}\right\}$ to $y$ in order to attain the sum $a_{i}$. This is, however, impossible since $\left\{a_{i}+l_{t}\right\}$ is an element of $y$.

We turn to show that after these first $k+1$ collapse steps are carried out, there remain no free $(k-1)$-faces in $X$. In order for a $(k-1)$-face $y$ to be free following the above collapses, $y$ has to be contained in exactly one of these $k+1$ collapsed faces. Since $\left\{a_{0}, \ldots, a_{k}\right\}$ is not an arithmetic progression, by Observation 3.1 , the intersection of any two of the $x^{(t)}$ contains at most $k-1$ elements. In particular there is no $(k-1)$-face that they both contain. Thus we have to consider only $(k-1)$-faces $y$ which are contained in one of the $x^{(t)}$ and exactly one more $k$-face.

It follows that $y$ must be of the form $x^{(t)} \backslash\left\{a_{j}+l_{t}\right\}$ for some $j$ and $t$. The sum of $y$ 's elements is $a_{t}-a_{j}-l_{t}$. If $y$ is contained as well in a $k$-face $z \in X_{a_{i}}$, then necessarily $z_{i}=z=y \cup\left\{a_{i}-a_{t}+a_{j}+l_{t}\right\}$. We are assuming that $y$ becomes free with the collapse of $x^{(t)}$, so there must be exactly one index $i$ for which $z_{i}$ is a legal $k$-face different from $x^{(t)}$. It follows that $x^{(t)}$ and $x^{(t)}+\left(a_{j}-a_{t}\right)$ must have $k$ elements in common. Again by Observation 3.1 this means that the elements in $x^{(t)}$ form an arithmetic progression, a contradiction. The proof of Theorem 1.3 is now complete.

\section{Example: Homology of $X_{\{0,1,3\}}$}

For a prime $p$ and an integer $n$ indivisible by $p$, let $U_{p, n}$ be the group of $n$th roots of unity in $\overline{\mathbb{F}_{p}}$. 
Proposition 4.1 Let $k=2, A=\{0,1,3\}$. Let $p$ be a prime and suppose that $n$ is coprime to $3 p$. Then

$$
h_{1}\left(X_{A} ; \mathbb{F}_{p}\right)=\frac{1}{3} \mid\left\{\{u, v\} \subset U_{p, n}-\{1\}: u \neq v \text { and } 1+u+v=0\right\} \mid .
$$

Proof Let $B=\{0, k, \ell\}$ with $0<k<\ell<n$, and let $u=\omega^{-k}, v=\omega^{-\ell}$. Then

$$
\begin{aligned}
\operatorname{det} M_{A, B} & =\operatorname{det}\left[\begin{array}{ccc}
1 & 1 & 1 \\
1 & u & v \\
1 & u^{3} & v^{3}
\end{array}\right] \\
& =u v^{3}-v u^{3}+u^{3}-u-v^{3}+v=(u-1)(v-1)(v-u)(u+v+1) .
\end{aligned}
$$

It follows that

$$
\operatorname{rk} M_{A, B}= \begin{cases}2, & 1+u+v=0 \\ 3, & \text { otherwise }\end{cases}
$$

Thus the Proposition follows directly from Theorem 1.

Corollary 4.2 Let $k=2$ and $A=\{0,1,3\}$. Let $p$ be a prime and suppose that $n=$ $p^{m}-1$ is coprime to 3 . Then

$$
h_{1}\left(X_{A} ; \mathbb{F}_{p}\right)= \begin{cases}\frac{n-1}{6}, & p=2, \\ \frac{n-2}{6}, & p=3 \\ \frac{n-4}{6}, & p>3 .\end{cases}
$$

Proof Clearly $\mathbb{F}_{p^{m}}^{*}=U_{p, n}$. Therefore, by Proposition 4.1,

$$
h_{1}\left(X_{A} ; \mathbb{F}_{p}\right)=\frac{1}{6}\left|\left\{u \in \mathbb{F}_{p^{m}}^{*}-\{1\}:-(1+u) \notin\{0,1, u\}\right\}\right| .
$$

The Corollary now follows since

$$
\left\{u \in \mathbb{F}_{p^{m}}^{*}-\{1\}:-(1+u) \notin\{0,1, u\}\right\}= \begin{cases}\mathbb{F}_{2^{m}}^{*}-\{1\}, & p=2 \\ \mathbb{F}_{3^{m}}^{*}-\{ \pm 1\}, & p=3 \\ \mathbb{F}_{p^{m}}^{*}-\left\{ \pm 1,-2,-\frac{1}{2}\right\}, & p>3\end{cases}
$$

\section{Concluding Remarks}

Theorem 1.1 provides an explicit description of the homology of the sum complex $X_{A}$ over fields of characteristic coprime to $n$. In particular, it follows via Chebotarëv's Theorem that if $n$ is prime, then $X_{A}$ is $\mathbb{Q}$-acyclic, i.e., $X_{A}$ is a $k$-hypertree. When $A$ is an arithmetic progression, $X_{A}$ was shown to be $k$-collapsible and in particular $\mathbb{Z}$-acyclic. One natural question is whether there exist other $A$ 's for which 
$X_{A}$ is $\mathbb{Z}$-acyclic. Kalai's $k$-dimensional Cayley's formula [1] suggests that most $k$-hypertrees are not $\mathbb{Z}$-acyclic. Likewise we conjecture that $X_{A}$ is not $\mathbb{Z}$-acyclic for most $(k+1)$-subsets $A \subset \mathbb{Z}_{n}$. One possible approach to the question of $\mathbb{F}_{p}$-acyclicity of $X_{A}$ for primes $p \nmid n$ is via the following reduction. Let $S_{\mathbb{F}}(A)$ be the $\mathbb{F}$-linear space of polynomials in $\mathbb{F}[x]$ spanned by the monomials $\left\{x^{a}: a \in A\right\}$. Theorem 1.1 then implies that $X_{A}$ is $\mathbb{F}_{p}$-acyclic iff $\operatorname{deg} \operatorname{gcd}\left(f(x), x^{n}-1\right) \leq k$ for all $0 \neq f(x) \in S_{\overline{\mathbb{F}_{p}}}(A)$.

\section{References}

1. Kalai, G.: Enumeration of $\mathbb{Q}$-acyclic simplicial complexes. Isr. J. Math. 45, 337-351 (1983)

2. Munkres, J.: Elements of Algebraic Topology. Addison-Wesley, Reading (1984)

3. Stevenhagen, P., Lenstra, H.W.: Chebotarëv and his density theorem. Math. Intell. 18, $26-37$ (1996) 